Supporting information

\title{
Multifunctional Conductive Hydrogel/Thermochromic Elastomer Hybrid Fibers with Core-Shell Segmental Configuration for Wearable Strain and Temperature Sensors
} Jingxuan Chen ${ }^{1,2}$, Hongji Wen ${ }^{1,3}$, Guoliang Zhang ${ }^{1,4}$, Fan Lei ${ }^{1,2}$, Qi Feng ${ }^{1,2}$, Yang Liu ${ }^{1,3}$, Xiaodong $\mathrm{Cao}^{1,2, *}$, Hua Dong ${ }^{1,2,3, *}$

1. Optimization of AMPS, AAm and rGO ratio in rGO-Poly(AMPS-co-AAm) hydrogel
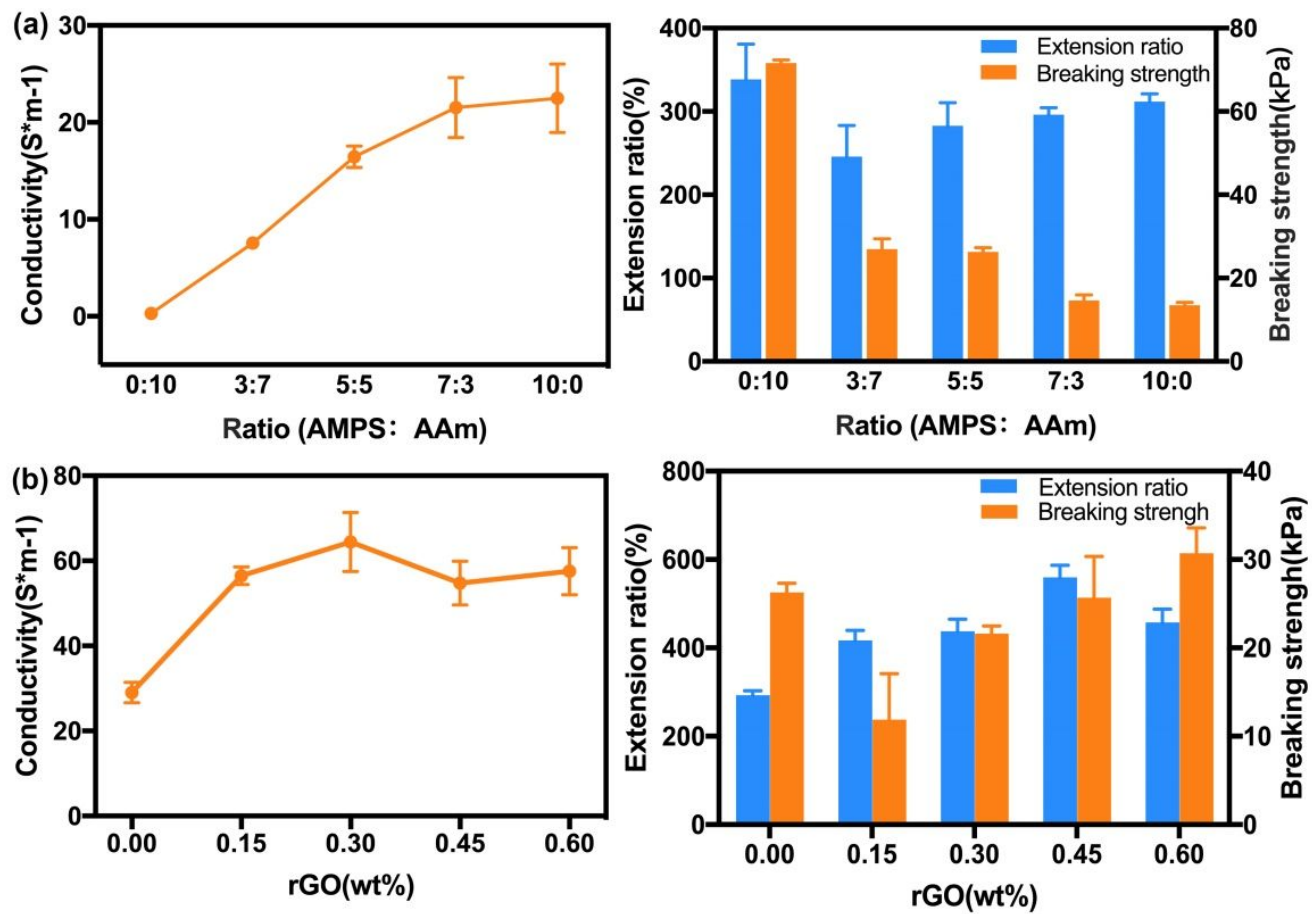

Figure S1. Optimization of AMPS, AAm and rGO ratio in rGO-Poly(AMPS-co-AAm) hydrogel

In order to figure out optimal AMPS/AAm ratio in rGO-Poly(AMPS-co-AAm) hydrogel, a series of samples with various AMPS/AAm ratios were tested with respect to conductivity and mechanical properties. As can be seen in Figure S1a, the hydrogel conductivity rises monotonously with the increase in APMS/AAm ratio. In contrast, maximum strain (or namely, extension rate) decreases sharply in the beginning and rises gradually with the AMPS/AAm ratio, whilst the breaking strength reduces with AMPS/AAm ratio. Taking 
both the conductivity and the mechanical strength into consideration, AMPS/AAm ratio is identified as 5:5 for rGO-Poly(AMPS-co-AAm) hydrogel. Figure S1b shows the variation of hydrogel conductivity as a function of rGO content in hydrogel. It is clear that the conductivity reaches saturation value when rGO content exceeds $0.15 \mathrm{wt} \%$. However, both extension rate and breaking strength don't show regular changes in the testing range of rGO content (0-0.6 wt \%). The optimal rGO content seems to be $0.3-0.45 \mathrm{wt} \%$. In our experiment, $0.3 \mathrm{wt} \%$ was used as the final rGO content in rGO-Poly(AMPS-co-AAm) hydrogel.

\section{Gauge factors (GF) of four acrylamide-based hydrogels}

Table S1. Gauge factors (GF) of four acrylamide-based hydrogels

\begin{tabular}{lccc}
\hline & $0-150 \%$ & $150-300 \%$ & $300-450 \%$ \\
\hline PAAm & 2.52 & 2.46 & N.A. \\
rGO-PAAm & 1.82 & 2.18 & N.A. \\
Poly(AMPS-co-AAm) & 3.07 & 5.50 & N.A. \\
rGO-Poly(AMPS-co-AAm) & 3.52 & 8.57 & 15.09 \\
\hline
\end{tabular}

*Data were obtained from Figure 1c. N.A. means the hydrogel samples were already broken before test.

\section{Mechanical characterization of rGO-Poly(AMPS-co-AAm) hydrogel}
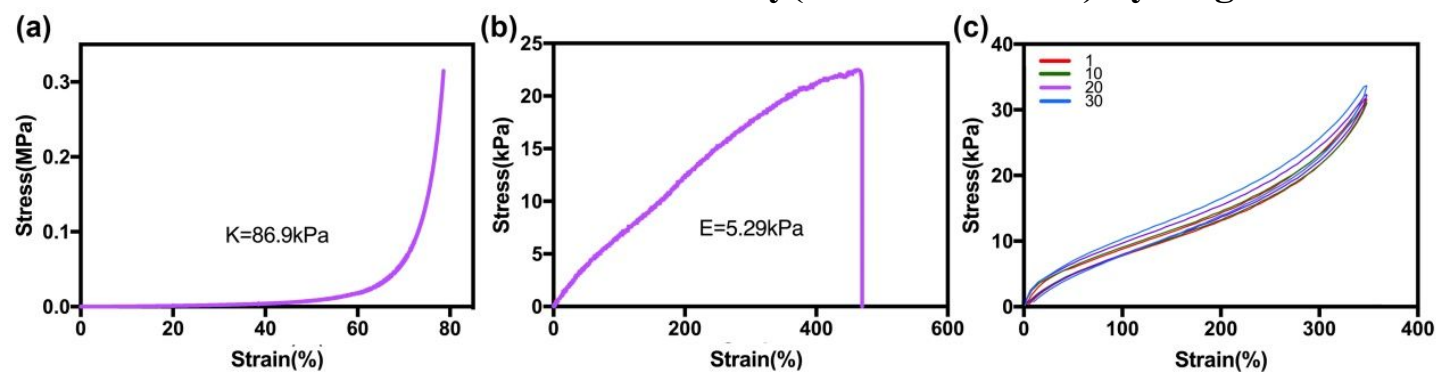

Figure S2. Mechanical characterization of rGO-Poly(AMPS-co-AAm) hydrogel: (a) compression test; (b)tensile test; (c) cyclic tensile test.

\section{SEM images of pure silicone rubber and thermochromic elastomer}


(a)

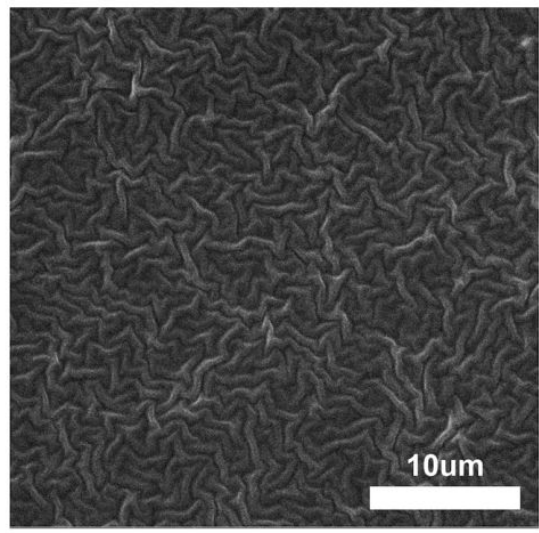

(b)

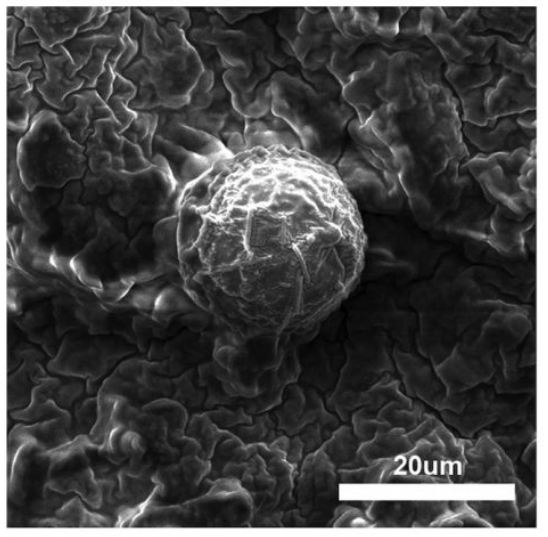

Figure S3. SEM images of (a) silicone rubber and (b) thermochromic elastomer composed of silicon rubber and thermochromic microcapsules

\section{Chemical compositions of thermochromic microcapsules}

\begin{tabular}{|c|c|c|}
\hline $\begin{array}{l}\text { Thermochromic } \\
\text { microcapsule }\end{array}$ & Chemical nature & wt $\%$ \\
\hline \multirow{5}{*}{ Blue- $-0^{\circ} \mathrm{C}$} & Melamine Formaldehyde Resin & $1 \sim 5 \%$ \\
\hline & 3-(4-Diethylaminophenyl)-3-(1-ethyl-2,methyl-indnl-3yl)phthalide & $2 \sim 10 \%$ \\
\hline & Butyl stearate & $5 \sim 15 \%$ \\
\hline & Ethyl palmitate & $5 \sim 15 \%$ \\
\hline & Meyhyl myristate & $40 \sim 60 \%$ \\
\hline \multirow{5}{*}{ Red- $35^{\circ} \mathrm{C}$} & Melamine Formaldehyde Resin & $1 \sim 5 \%$ \\
\hline & 3-N-p-tolyl-N-ethylamino-7-methyl-fluoran & $2 \sim 10 \%$ \\
\hline & 4,4-Isopropylidenediphenol & $5 \sim 15 \%$ \\
\hline & Methyl stearate & $20 \sim 40 \%$ \\
\hline & 1-Tetradecanol & $20 \sim 40 \%$ \\
\hline \multirow{5}{*}{ Green- $55^{\circ} \mathrm{C}$} & Melamine Formaldehyde Resin & $1 \sim 5 \%$ \\
\hline & 3,6-Dimethoxyfluoran & $1 \sim 5 \%$ \\
\hline & 3-(4-Dimethylaminophenyl)-3-(1-ethyl-2,methylindnl-3yl)phthalide & $1 \sim 5 \%$ \\
\hline & 4,4-Isopropylidenediphenol & $5 \sim 15 \%$ \\
\hline & 1-Octadecanol & $50 \sim 80 \%$ \\
\hline
\end{tabular}




\section{Color circle showing complementary color}

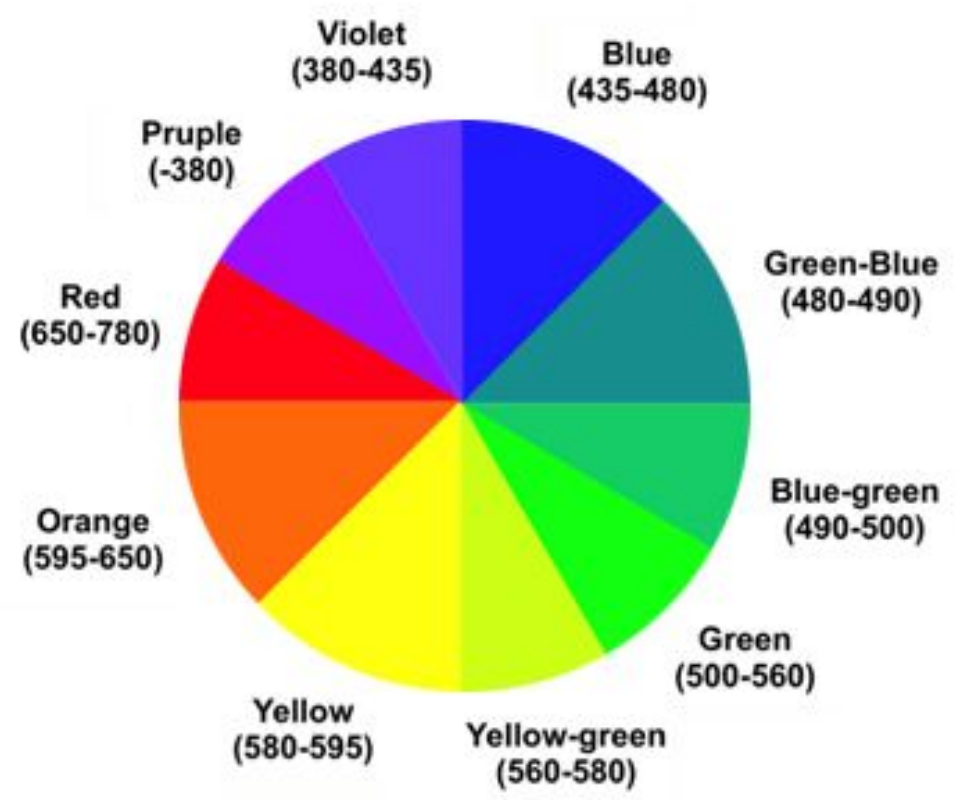

Figure S4. Color circle showing complementary colors in visible region

\section{Selection of shell material for multifunctional hybrid fibers}
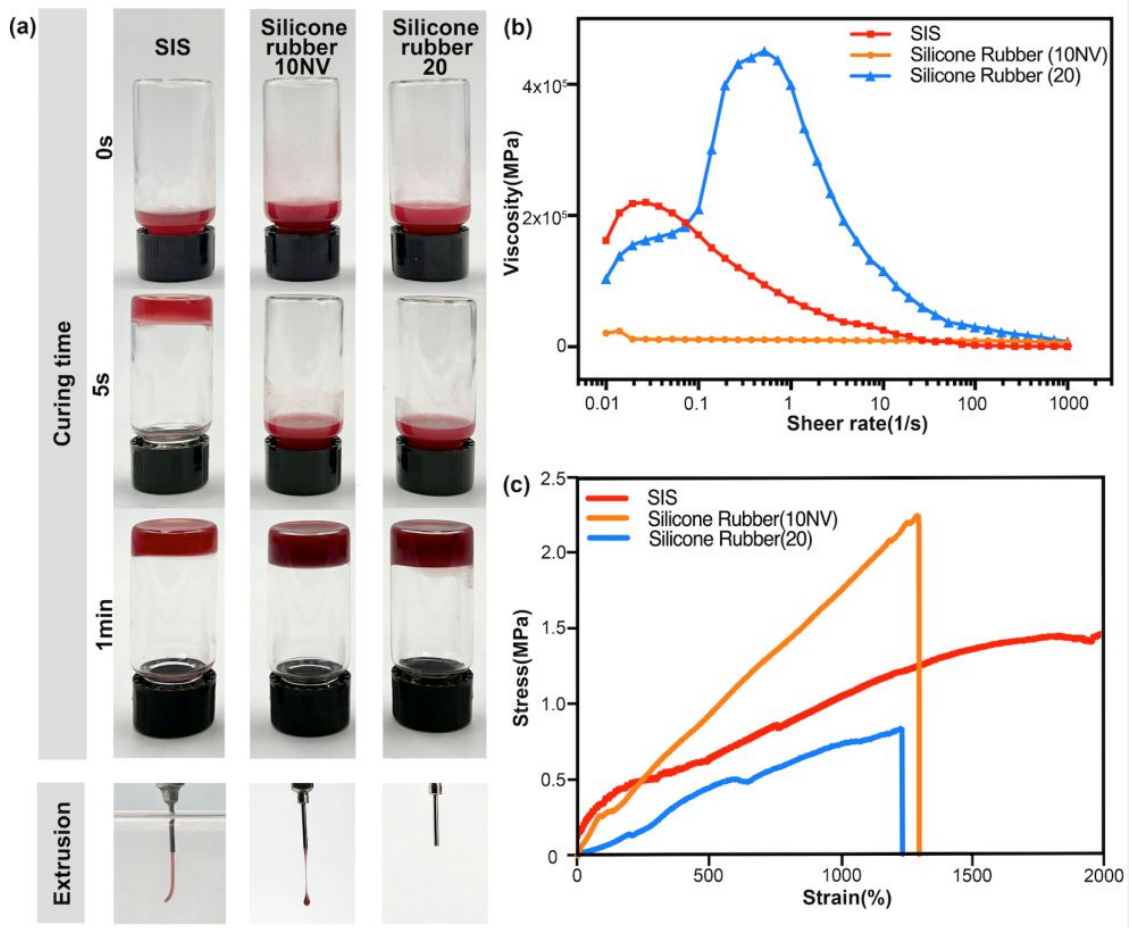

Figure S5. Selection of shell material for multifunctional hybrid fibers: (a) curing time and extrusion of SIS, silicone rubber 10NV (Smooth-on, Inc) and silicone rubber 20 (Smooth-on, Inc). For the convenience of observation, red dyes were added into the test samples; (b) rheological and (c) tensile tests of SIS, Silicone 
rubber $10 \mathrm{NV}$ and Silicone rubber 20.

In order to realize continuous extrusion of multifunctional fibers with core-shell configuration, the shell material needs to be cured quickly after extrusion and the resulting shell layer should be highly elastic. Three materials, i.e., polystyrene-block-polyisoprene -block-polystyrene (SIS), silicone rubber $10 \mathrm{NV}$ and silicone rubber 20, were compared in terms of their curing time, rheological and tensile properties. It can be seen in Figure S5a, SIS dissolved in $\mathrm{CH}_{2} \mathrm{Cl}_{2}$ solution can be cured in $5 \mathrm{~s}$ when exposed to alcohol and the other two materials are not cured until 1 min. Moreover, only SIS can be extruded successfully, mainly due to the medium viscosity as shown in Figure S5b. Figure S5c reveals that SIS owns the highest tensile modulus in all the three materials. As a result, SIS was chosen as the shell material.

\section{Water evaporation from the core-shell conductive hydrogel fiber and naked conductive hydrogel fiber}

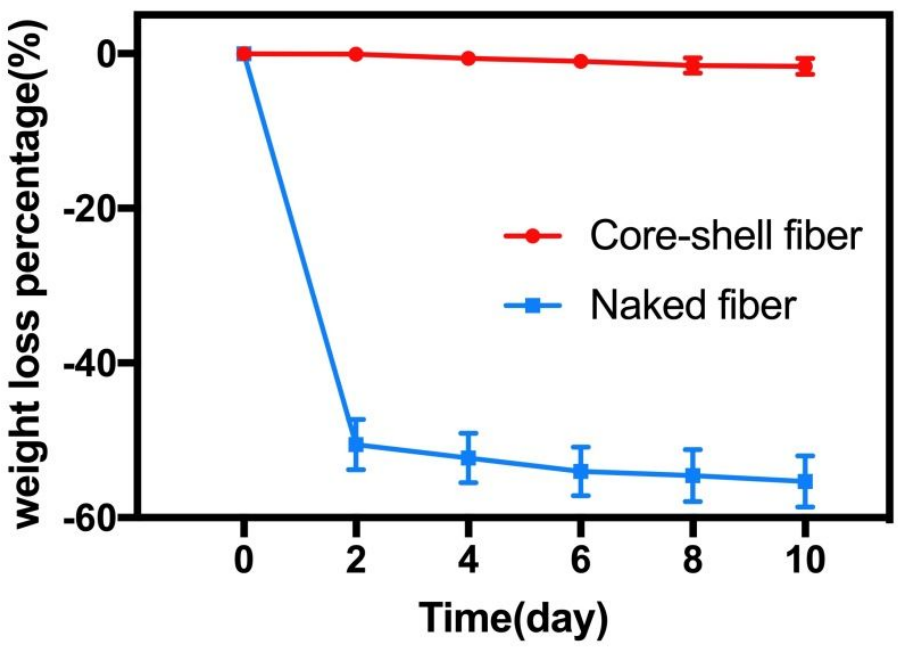

Figure S6. Weight loss percentage of core-shell fiber and naked fiber as a function of storage time

In addition to act as a good insulator, another important role of shell layer is to prevent water evaporation of conductive hydrogel so that its resistance can maintain constant. Figure S6 compares the weight loss of core-shell fiber and naked fiber as a function of storage time. As can be seen, the naked conductive hydrogel fiber loses most of water $(\sim 50.43 \mathrm{wt} \%)$ in the first two days and continues declining slowly, whilst the coreshell conductive fiber only loses $\sim 1.65 \mathrm{wt} \%$ of water after ten days of storage. This effect can be further enhanced by coating a thin parylene layer on the outer surface of shell layer. ${ }^{1}$ 


\section{Photos of thermochromic elastomer fiber (top) and their cross sections before and}

\section{after post treatment}

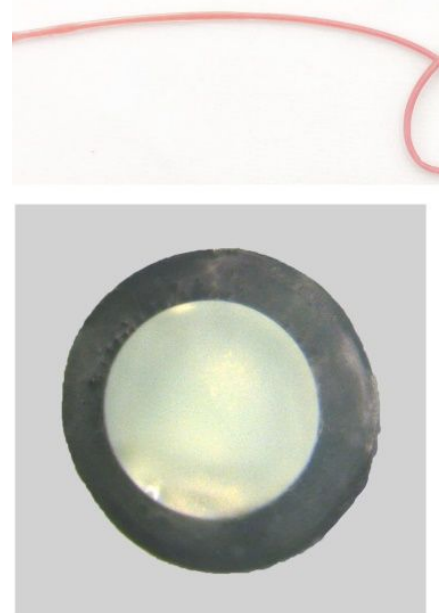

before heat-treatment
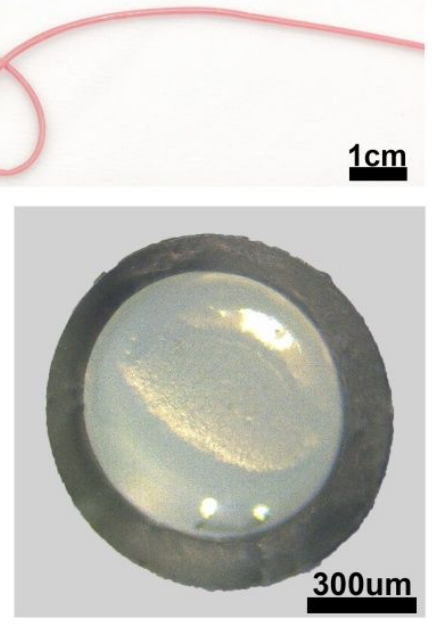

after heat-treatment

Figure S7. Photos of thermochromic elastomer fiber (top) and cross sections of thermochromic elastomer fibers with unsolidified core (bottom left) and solidified cores (bottom right). Note that the fibers before and after heat-treatment were not the same one. After cutting the thermochromic elastomer fiber before heat-treatment, the unsolidified thermochromic elastomer prepolymer would flow out from the fiber, which was not suitable for the following heat-treatment. In our experiment, another thermochromic elastomer fiber was heated and then cut for observation.

\section{Mechanical characterization of conductive hydrogel fiber and thermochromic elastomer fiber}
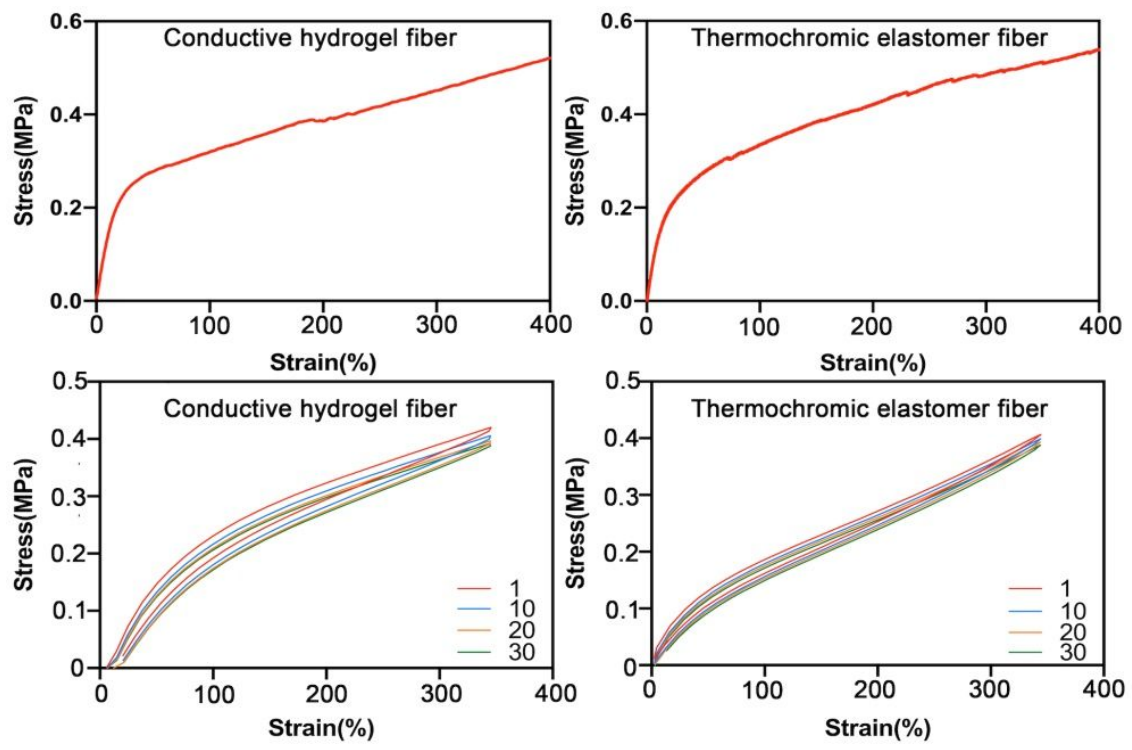
Figure S8. Mechanical characterization of conductive hydrogel fiber and thermochromic elastomer fiber

\section{Conductive hydrogel/thermochromic elastomer hybrid fibers as wearable strain and temperature sensors to monitor shoulder and elbow motions}
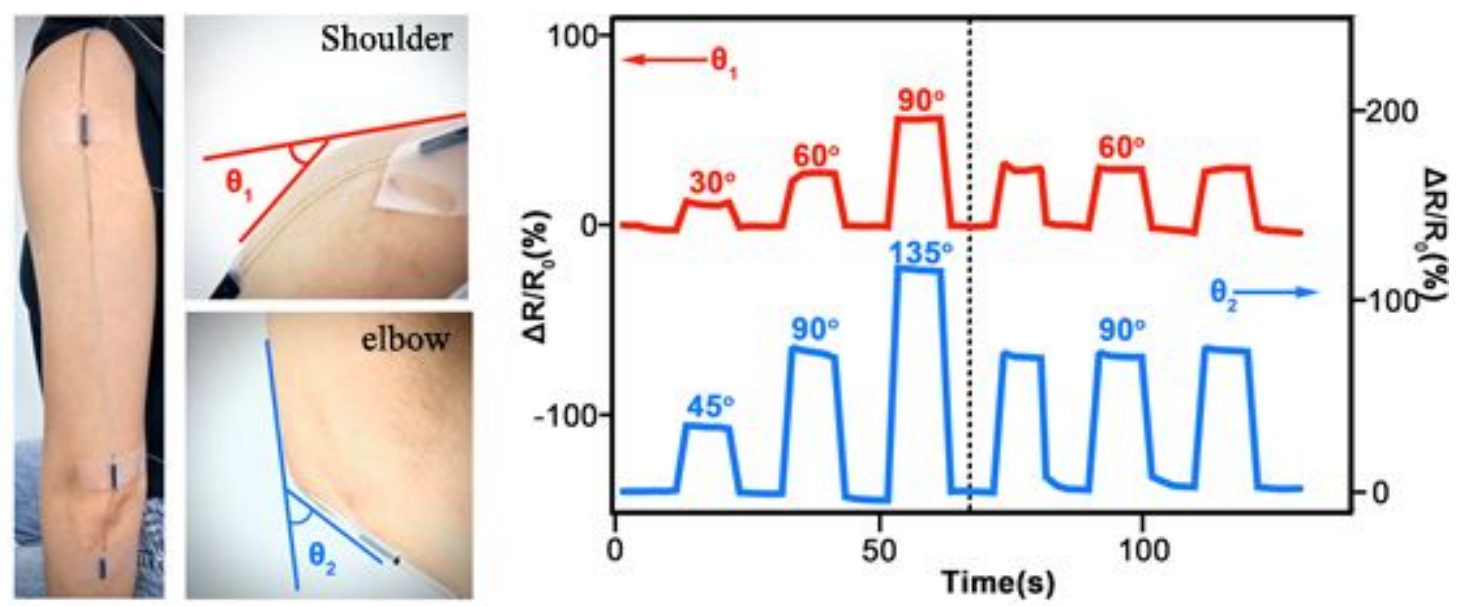

Figure S9. Conductive hydrogel/thermochromic elastomer hybrid fibers as wearable strain and temperature sensors to monitor shoulder and elbow motions.

\section{Images of GO solution and rGO solution}

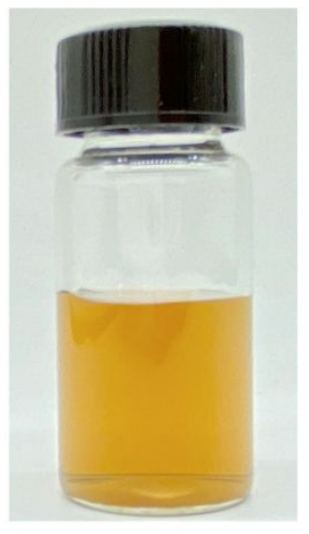

GO

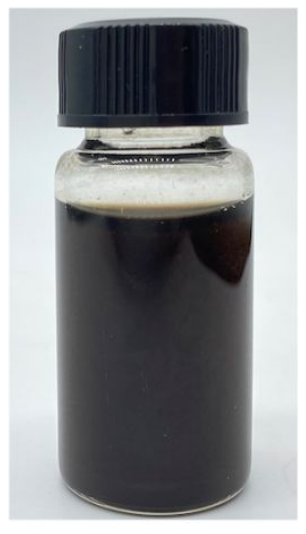

rGO

Figure S10. Images of GO solution (raw material) and rGO solution (after adding DA into GO solution)

\section{References:}

(1) Yasuda, H. Plasma polymerization for protective coatings and composite membranes. Journal of membrane science 1984, 18, 273-284. 